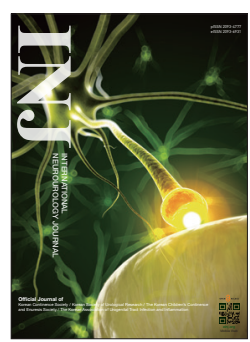

\title{
Expected Next-Generation Drugs
}

\author{
Khae Hawn Kim (iD https://orcid.org/0000-0002-7045-8004 \\ Department of Urology, Gachon University Gil Hospital, Gachon University of Medicine and Science, Incheon, Korea \\ E-mail:kimcho99@gilhospital.com
}

Recently, the world has been covered with the wave of the fourth industrial revolution, but, according to industry, the concept is so diverse that it is still difficult to define the fourth industrial revolution as one entity. Among the possible delineations, the emerging fourth industrial revolution will probably be the next generational revolution across the whole bio-medical industry, fusing knowledge-intensive sciences such as physics, chemistry, life sciences, and engineering. These new medical technology streams combined with artificial intelligence, artificial organ development by 3-dimensional printing technology, nanotechnology, and the development of new drugs are now the most representative field.

Among these scientific endeavors, the development of new drugs is also a traditional science and an industry that has continuously carried on from the past, but it is also a high-tech industry that has attracted both industrial and medical attention. New drug development requires years of continuous investment of pharmaceuticals, efforts by researchers, and, if possible, national support. In addition, overall trust for the pharmaceutical industry is also a very important factor. because of the following possible problems in new drug development. First, it may be used as a monopoly on intellectual property rights and a way to maintain market dominance for capital and market dominant pharmaceuticals, even if there is no definite superior effect compared with existing medicines. Second, because pharmaceutical companies have invested a great deal of capital, there could be a tendency to hide the results of clinical trials, even though the results are unfavorable, in order to avoid immediate responsibility. In terms of these problems, the research ethics and scholarly conscience of researchers who lead the development of new drugs are of increasing importance and, after all, enhancing the level of consciousness of society as a whole to increase the conscience level of individual scholars also plays a major factor.

Despite some of these concerns, the successful development of a new drug could have profound benefits. As noted in the article of this issue [1], these benefits span to patients, researchers, and nations, as well as to individual pharmaceutical companies. This is why we are more and more involved in new drug development.

As science advances, technological achievements have been made in finding and synthesizing candidate substances of new drugs. However, the share of new breakthrough drugs in the market is not very high. In this respect, it is very encouraging that several drugs such as mirabegron, phosphodiesterase type 5 inhibitor, and botulinum toxin A have been successfully launched at a similar time in the clinical field of urology. However, though the development of new drug candidate materials is important, the interest in drug formulas, action method and time, and drug delivery systems is also growing, and it is very encouraging that these drug action mechanisms have been tried in addition to traditional drug action methods. Generally, although not mentioned in detail in the article, luteinizing hormone-releasing hormone agonists, vasopressin and diverse combination therapy, and other methods for modifying drug delivery methods, such as vaccines, extended release tablets, and intraprostatic injection therapy, are important attempts to dramatically expand the span of voiding related symptoms. 


\section{INJ}

Overall, this article is a meaningful review about the aspects of new drug development in relation to voiding symptoms that are very common in the urology field, where the interest of the fourth industrial revolution is peaking.

- Conflict of Interest: No potential conflict of interest relevant to this article was reported.

\section{REFERENCE}

1. Chung KJ, Chung BI. Expected next-generation drugs under development in relation to voiding symptoms. Int Neurourol J 2017; 21:97-101. 